\title{
Tellurium-Doped, Mesoporous Carbon Nanomaterials as Transparent Metal-Free Counter Electrodes for High-Performance Bifacial Dye-Sensitized Solar Cells
}

\author{
Chang Ki Kim, Jung-Min Ji, Haoran Zhou, Chunyuan Lu and Hwan Kyu Kim * \\ Global GET-Future Lab. \& Department of Advanced Materials Chemistry, Korea University, 2511 Sejong-ro, \\ Sejong 339-700, Korea; rornfl1982@naver.com (C.K.K.); manbbong@korea.ac.kr (J.-M.J.); \\ zhouhaoran@naver.com (H.Z.); lcy99168@gmail.com (C.L.) \\ * Correspondence: hkk777@korea.ac.kr
}

Received: 21 November 2019; Accepted: 17 December 2019; Published: 20 December 2019

\begin{abstract}
Tellurium-doped, mesoporous carbon nanomaterials with a relatively high doping level were prepared by a simple stabilization and carbonization method in the presence of a tellurium metalloid. A transparent counter electrode (CE) was prepared using tellurium-doped, mesoporous carbon (TeMC) materials, and was directly applied to bifacial, dye-sensitized solar cells (DSSCs). To improve the performance of the bifacial DSSC device, CEs should have outstanding electrocatalytic activity, electrical conductivity, and electrochemical stability, as well as high transparency. In this study, to make transparent electrodes with outstanding electrocatalytic activity and electrical conductivity, various TeMC materials with different carbonization temperatures were prepared by simple pyrolysis of the polyacrylonitrile-block-poly (n-butyl acrylate) (PAN-b-PBA) block copolymer in the presence of the tellurium metalloid. The electrocatalytic activity of the prepared TeMC materials were evaluated through a dummy cell test, and the material with the best catalytic ability was selected and optimized for application in bifacial DSSC devices by controlling the film thickness of the CE. As a result, the bifacial DSSC devices with the TeMC CE exhibited high power conversion efficiencies (PCE), i.e., $9.43 \%$ and $8.06 \%$ under front and rear side irradiation, respectively, which are the highest values reported for bifacial DSSCs to date. Based on these results, newly-developed transparent, carbon-based electrodes may lead to more stable and effective bifacial DSSC development without sacrificing the photovoltaic performance of the DSSC device.
\end{abstract}

Keywords: dye-sensitized solar cells; counter electrodes; bifacial devices; tellurium-doped mesoporous carbon; transparency

\section{Introduction}

Dye-sensitized solar cells (DSSCs), which can directly convert solar energy into electrical energy, are seen as promising energy conversion devices due to their high power conversion efficiency (PCE), easy fabrication process, and environmentally-friendly nature [1-4]. In addition to these advantages, DSSCs have favorable characteristics such as their color and transparency, as well as their efficiency under ambient light conditions (i.e., for indoor operation) [5]. Therefore, these devices could be applied to building-integrated photovoltaics (BIPVs), which can utilize external and internal lights [6,7].

Conventional DSSC devices consist of the three following main components: a dye-absorbed $\mathrm{TiO}_{2}$ photoanode, a redox couple (iodine: $\mathrm{I}^{-} / \mathrm{I}_{3}^{-}$, cobalt: $\left.\mathrm{Co}(\mathrm{bpy})_{3}{ }^{2+/ 3+}\right)[8-10]$, and a counter electrode (CE). Among these main components, the role of the CE in DSSC devices is for electron transfer from the external circuit to the electrode surface for the reduction of the oxidized redox species [11-13]. Therefore, CEs should have high electrical conductivity and electrocatalytic activity for high-performance DSSC 
devices. Generally, platinum (Pt) has been widely used as a CE in DSSCs because of its outstanding electrical conductivity and electrocatalytic activity. Nevertheless, Pt-based CEs cannot be applied to large-scale applications because of their high cost and limited supply. Therefore, it is necessary to develop novel materials at low cost, and with high electrocatalytic activity and electrical conductivity in order to replace Pt. In recent years, various candidate materials such as carbonaceous materials [14-17], polymers $[18,19]$, metal compounds $[20,21]$ and their combinations $[22,23]$ have been developed to this end. Among them, carbon materials are used in many fields, such as DSSCs [24,25], fuel cells [26], supercapacitors [27], batteries [28], and sensors [29], because of their high electrical conductivity, high specific surface area, and low cost.

In this study, we prepared carbon-based CEs using tellurium-doped, mesoporous carbon (TeMC) materials to replace Pt-based CEs, and applied them to the CEs in DSSCs. Previous studies have already reported that Te-doped carbon materials can be prepared and applied as the CEs of a DSSC, showing outstanding electrocatalytic performance for cobalt and iodine electrolytes [30]. However, due to the low doping level of tellurium in the carbon structure, it did not perform significantly better than Pt. Therefore, we improved the electrocatalytic activity by increasing the doping level of tellurium; at the same time, a transparent electrode was prepared while maintaining its high electrocatalytic activity, which was introduced into the bifacial DSSC device. To make bifacial DSSC devices, the CE and electrolyte must be transparent, because irradiated light from the rear side passes through the CE and the electrolyte before finally reaching the photoanode. Therefore, the CE should have the following characteristics for use in bifacial DSSCs: (i) high electrocatalytic activity for the reduction of oxidized redox couples compared with Pt-based CEs, (ii) high electrical conductivity for the transfer of electrons from the external circuit to the electrode surface, (iii) high transmittance to improve the performance of rear-side irradiation, and (iv) high electrochemical stability for industrial applications [31-33]. In this study, to make the transparent electrode while maintaining its electrocatalytic activity and electrical conductivity, we prepared TeMC materials with different carbonization temperatures. According to the carbonization temperature, as-prepared TeMC materials have different Te doping levels and electrical conductivities; therefore, they have different performance when used as CEs. The electrocatalytic abilities of the prepared TeMC materials were evaluated through a dummy cell test, and the material with the best catalytic ability was selected for application in bifacial DSSC devices. To prepare the transparent $\mathrm{CE}$, the selected a TeMC material that was optimized by controlling the film thickness when preparing the $\mathrm{CE}$. The optimized TeMC CE had excellent electrocatalytic activity, electrical conductivity, and electrochemical stability, as well as high transmittance, and it showed excellent performance when applied to the bifacial DSSC device.

\section{Experimental Section}

\subsection{Materials}

N-butyl acrylate, $\alpha, \alpha^{\prime}$-azoisobutyronitrile, $\mathrm{CuBr}_{2}$, tris(2-pyridylmethyl)amine, ethyl $\alpha$-bromoisobutyrate, acrylonitrile, 2,2'-bipyridyl, $\mathrm{CuCl}, \mathrm{Al}_{2} \mathrm{O}_{3}$, dimethylformamide, dimethyl sulfoxide, ethanol, toluene, methanol, and tellurium were all obtained from Sigma-Aldrich (Seoul, Korea).

\subsection{Preparation of Tellurium-Doped, Mesoporous Carbon (TeMC) Materials}

The PAN-b-PBA (polyacrylonitrile-block-poly (n-butyl acrylate) copolymer (2 g) was physically mixed with $1 \mathrm{~g}$ of tellurium powder and placed into a quartz furnace. The mixture was stabilized at $280{ }^{\circ} \mathrm{C}$ for $2 \mathrm{~h}$ under an air atmosphere (heating rate: $5{ }^{\circ} \mathrm{C} / \mathrm{min}$ ) and then carbonized at three different temperatures $\left(700,800\right.$, and $900{ }^{\circ} \mathrm{C}$ ) for $1 \mathrm{~h}$ under a nitrogen atmosphere (heating rate: $5{ }^{\circ} \mathrm{C} / \mathrm{min}$ ). The final products were washed with $1 \mathrm{M} \mathrm{HCl}$ and $3 \mathrm{M} \mathrm{KOH}$ aqueous solution to remove the unreacted tellurium. 


\section{Results and Discussion}

In a previous study, we successfully prepared the polyacrylonitrile-block-poly(n-butyl acrylate) (PAN-b-PBA) block copolymer using the atom transfer radical polymerization method, and directly used it to make tellurium-doped porous carbon materials [24,30]. The prepared carbon materials were used as a CE in DSSCs, and showed outstanding performance due to their mesoporous structure and many defect sites. In this study, to increase the electrocatalytic activity, we improved the doping level of tellurium by a simple stabilization and carbonization method in the presence of a tellurium metalloid. The preparation method is as follows (see Figure 1). First, the prepared block copolymer (PAN-b-PBA), according to a previous report [30], and tellurium metalloid were physically mixed; then, the mixture was put into a quartz furnace. To prepare the porous carbon materials, the mixture was stabilized under an air atmosphere and carbonized under a $\mathrm{N}_{2}$ atmosphere. After these processes, to remove the unreacted elemental tellurium, the resultant materials were washed with $1 \mathrm{M} \mathrm{HCl}$ and 3 $\mathrm{M} \mathrm{KOH}$ aqueous solution. To investigate the effect of temperature during the preparation process, we prepared the TeMC materials at different temperatures $\left(700,800\right.$, and $\left.900{ }^{\circ} \mathrm{C}\right)$ in the carbonization process. The preparation method of TeMC materials is described in the Experimental section.

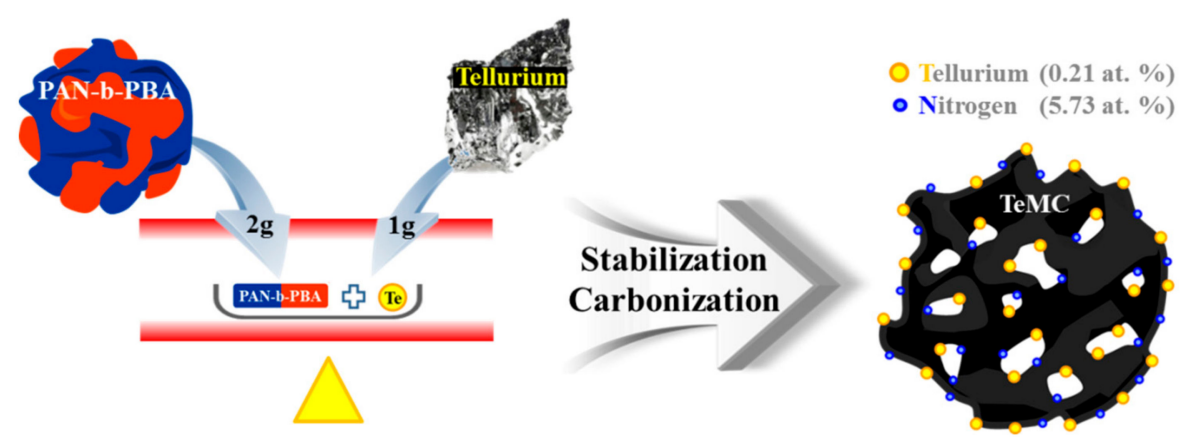

Figure 1. Schematic illustration of the preparation method of tellurium-doped, mesoporous carbon (TeMC) materials.

Field emission scanning electron microscopy (FE-SEM) (Hitachi, Japan) and high-resolution transmission electron microscopy (HR-TEM) (JEOL Ltd., Japan) analyses were carried out to confirm the surface morphology and porous structure of the TeMC materials. Figure 2 shows FE-SEM (Figure 2a,c,e) and HR-TEM (Figure 2b,d,f) images of the as-prepared TeMC materials with different carbonization temperatures. As shown in the SEM images, all of the prepared TeMC materials have an interconnected porous nature with three-dimensional, bicontinuous structures. These structures are more clearly identifiable in the TEM images. As shown, all of the TeMC materials have uniform pore structures that are homogeneously dispersed in the carbon framework. 

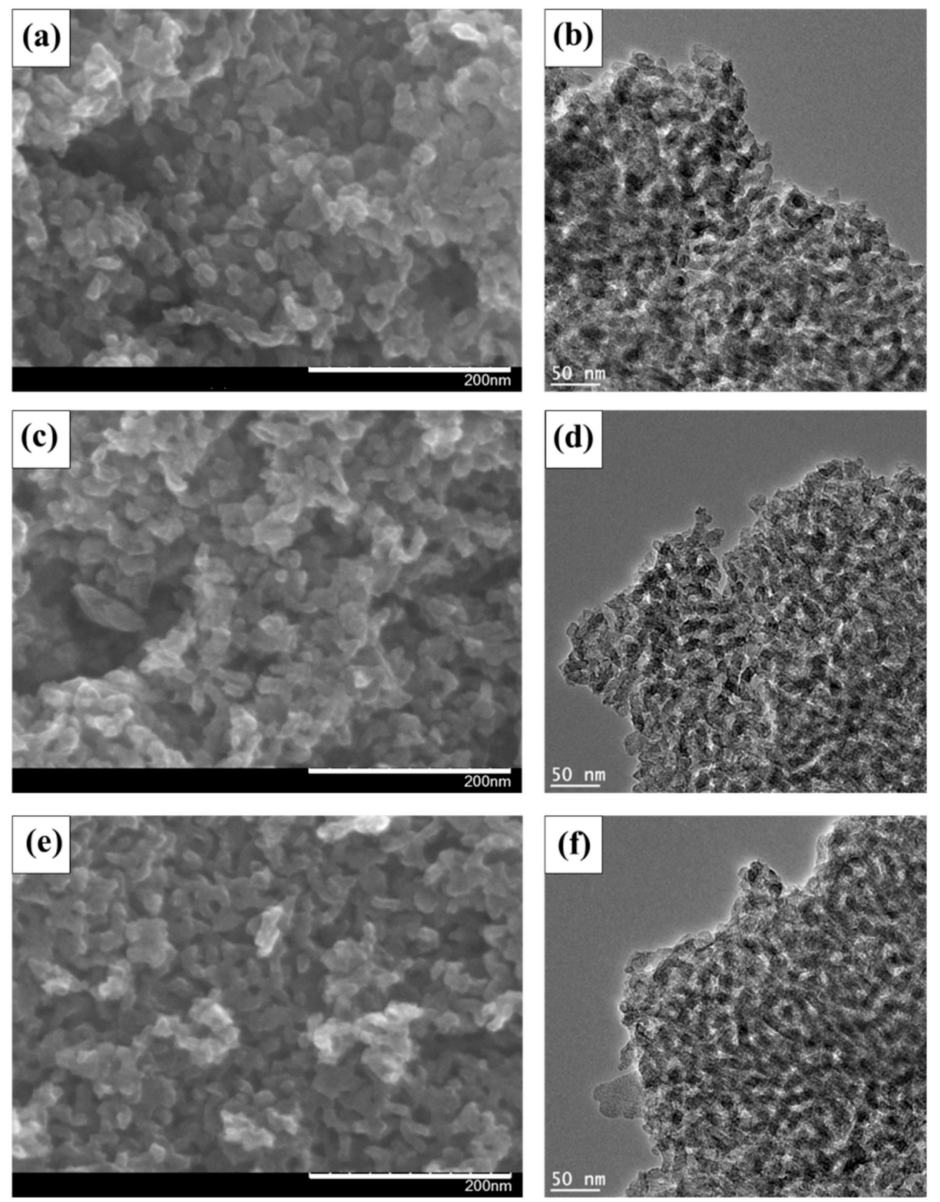

Figure 2. Field emission scanning electron microscopy (a,c,e) and high-resolution transmission electron microscopy $(\mathbf{b}, \mathbf{d}, \mathbf{f})$ images of the prepared TeMC materials with different carbonization temperatures $(\mathbf{a}, \mathbf{b}): 700^{\circ} \mathrm{C} ;(\mathbf{c}, \mathbf{d}): 800^{\circ} \mathrm{C} ;(\mathbf{e}, \mathbf{f}): 900^{\circ} \mathrm{C}$.

To understand the porous structure more clearly, nitrogen adsorption-desorption isotherm measurements were carried out. Figure 3a shows the nitrogen sorption isotherms data for the as-prepared TeMC materials; the corresponding porosity properties are summarized in Table 1 . The shape of the isotherms can be distinguished into six types according to the International Union of Pure and Applied Chemistry (IUPAC) classification [34]. Among them, the isotherm data of the prepared TeMC materials, as shown in Figure 3a, correspond to type IV with an H1 hysteresis loop, which represents a well-developed mesopore and interconnected pore structure. The specific surface area and pore volumes are calculated and summarized in Table 1. The specific surface areas of the TeMC materials with different carbonization temperatures are $399.68,455.05$, and $526.79 \mathrm{~m}^{2} \mathrm{~g}^{-1}$, and the total pore volumes are $0.80,0.78$, and $0.87 \mathrm{~cm}^{3} \mathrm{~g}^{-1}$. As a result, the specific surface area increased with increasing temperature, and the total pore volume increased accordingly. In particular, the TeMC-900 material has a higher specific surface area and micropore volume than that of other materials (TeMC-700 and TeMC-800), because the carbon surface rapidly decomposed at high temperatures, forming micropores. Therefore, the specific surface area increased with increasing carbonization temperature. Well-developed mesoporous structures and high specific surface areas within the carbon network give rise to more efficient diffusion of electrolyte ions and contact with these ions. Figure $3 \mathrm{~b}$ shows the wide-angle x-ray diffraction (XRD) spectra of the TeMC materials. All of the materials have two typical broad peaks at $25^{\circ}$ and $43^{\circ}$, which were assigned to the [002] and [100] diffraction planes [35]. These XRD peaks indicate that the TeMC materials have an amorphous and disordered carbon structure with many defect sites. 

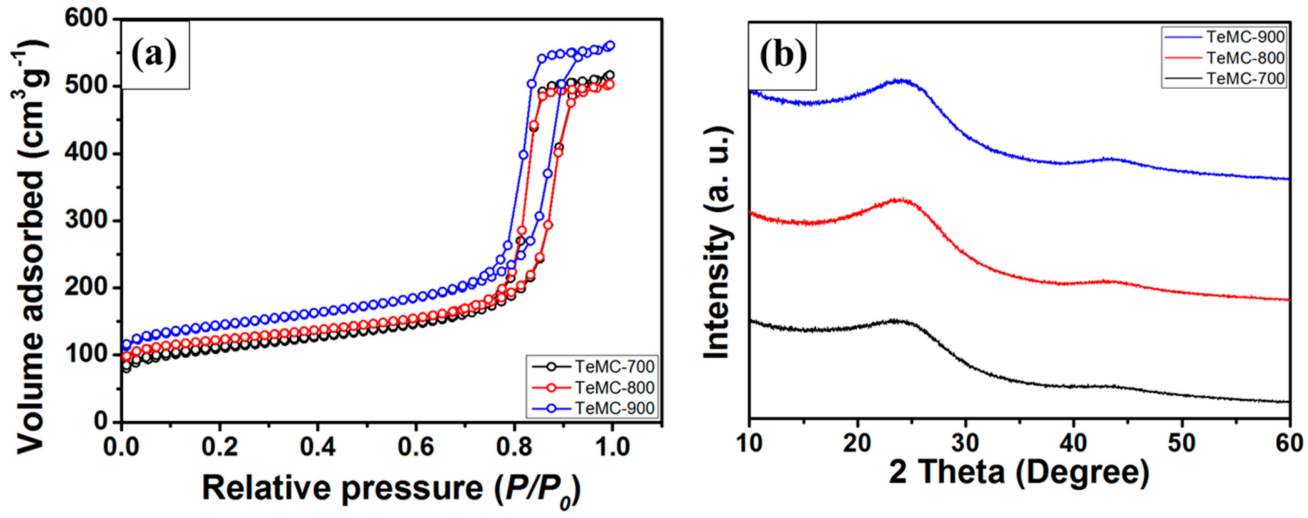

Figure 3. (a) Nitrogen adsorption-desorption isotherm data and (b) wide-angle x-ray diffraction spectra of TeMC materials.

Table 1. Porosity properties of TeMC materials with different carbonization temperatures.

\begin{tabular}{cccccc}
\hline Sample & $\begin{array}{c}S_{\text {BET }} \\
\left(\mathbf{m}^{\mathbf{2}} \mathbf{g}^{\mathbf{1}}\right)\end{array}$ & $\begin{array}{c}\boldsymbol{V}_{\text {total }} \\
\left(\mathbf{c m}^{\mathbf{3}} \mathbf{g}^{\mathbf{- 1}}\right)\end{array}$ & $\begin{array}{c}\boldsymbol{V}_{\text {micro }} \\
\left(\mathbf{c m}^{\mathbf{3}} \mathbf{g}^{\mathbf{- 1}}\right)\end{array}$ & $\begin{array}{c}\boldsymbol{V}_{\text {meso }} \\
\left(\mathbf{c m}^{\mathbf{3}} \mathbf{g}^{\mathbf{- 1}}\right)\end{array}$ & $\begin{array}{c}\text { Pore Size } \\
(\mathbf{n m})\end{array}$ \\
\hline TeMC-700 & 399.68 & 0.80 & 0.08 & 0.73 & 12 \\
TeMC-800 & 455.05 & 0.78 & 0.11 & 0.68 & 12 \\
TeMC-900 & 526.79 & 0.87 & 0.12 & 0.75 & 11 \\
\hline
\end{tabular}

$S_{\mathrm{BET}}$ : $\mathrm{BET}$ surface area, $V_{\text {total }}$ : total pore volume, $V_{\text {micro }}$ : micropore volume, $V_{\text {meso }}$ : mesopore volume.

To further investigate the structural characterization of the prepared TeMC samples, Raman spectroscopy was used. As shown in Figure 4, three different peaks were observed at 1350, 1590, and $1510 \mathrm{~cm}^{-1}$, corresponding to the $D$ (disordered graphitic structure or defective graphite), $G$ (graphitic structure), and $A$ (amorphous structure) bands. The relative intensity ratio of the $D$ to $G$ band $\left(I_{D} / I_{G}\right)$ is a useful tool for evaluating the number of defects in the carbon framework. As calculated in Figure 4, the $I_{D} / I_{G}$ ratio decreased with increasing carbonization temperature due to the decomposition of the defect sites, which are mainly distributed at the edges of the carbon frameworks. As shown in Figure $\mathrm{S} 1$, the electrical conductivity of TeMC materials increased with increasing carbonization temperature because of the decrease in the number of defect sites.
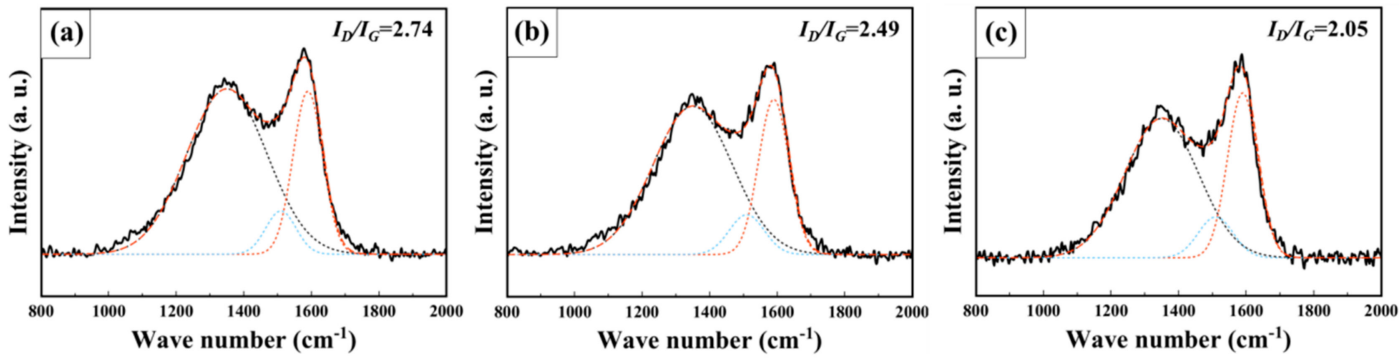

Figure 4. Raman spectra of TeMC materials with different carbonization temperatures (a): 700, (b): 800, (c): $900{ }^{\circ} \mathrm{C}$.

To confirm the elemental composition and bonding configurations of the elements in the carbon materials, $x$-ray photoelectron spectroscopy (XPS) was carried out. As shown in Figure 5 and Table 2, all TeMC materials mainly consisted of carbon, nitrogen, oxygen, and tellurium. After the carbonization process, tellurium was introduced into the carbon structure; XPS analysis confirmed that tellurium was present. As shown in Table 2, according to the increase in carbonization temperature, the contents of Te and $\mathrm{N}$ elements were reduced. More specifically, to understand the bonding configuration of the as-prepared carbon materials, a high-resolution XPS spectrum was analyzed. As shown in Figure S2, 
the C1s spectra (Figure S2a-c) can be deconvoluted into five different peaks at around 283.3, 284.6, 285.7, 286.8, and $288.5 \mathrm{eV}$, corresponding to $\mathrm{C}-\mathrm{Te}$, graphitic carbon $\left(\mathrm{sp}^{2}\right.$ and $\left.\mathrm{sp}^{3}\right), \mathrm{C}-\mathrm{O}, \mathrm{C}=\mathrm{Te}$, and $\mathrm{C}=\mathrm{O}$, respectively [32]. Deconvolution of the O1s spectra (Figure S2d-f) shows three major peaks at 530.8 $(\mathrm{Te}-\mathrm{O}), 531.8(\mathrm{C}=\mathrm{O})$, and $533.0 \mathrm{eV}(\mathrm{C}-\mathrm{O})$ [36]. The high-resolution N1s XPS spectra (Figure S2g-i) shows four types of nitrogen species at around 398.2 (pyridinic- $N$ ), 400.1 (pyrrolic- $N$ ), 401.2 (quaternary- $N$ ), and $402.4 \mathrm{eV}$ (pyridinic- $N$-oxide) [37]. The Te3d spectra were also deconvoluted into four peaks with binding energies of $573.8,575.7,584.2$, and $586.2 \mathrm{eV}$, assigned to the oxidized tellurium (575.7 and 586.2 $\mathrm{eV}$ ) and the C-Te peak (573.8 and $584.2 \mathrm{eV}$ ) [32]. From these high-resolution XPS results, we know that the elemental tellurium is well incorporated into the carbon structure. As shown in Figure S3, the pyridinic and pyrrolic nitrogen are mainly located at the edge side, but quaternary nitrogen is mostly located at the center side. Therefore, when the carbonization temperature is increased, the pyridinic and pyrrolic nitrogen are rapidly decomposed because they are located at the edge side. Figure S4 shows elemental mapping data confirming the dispersity of each element in the carbon framework. All elements were homogeneously dispersed in the carbon framework.

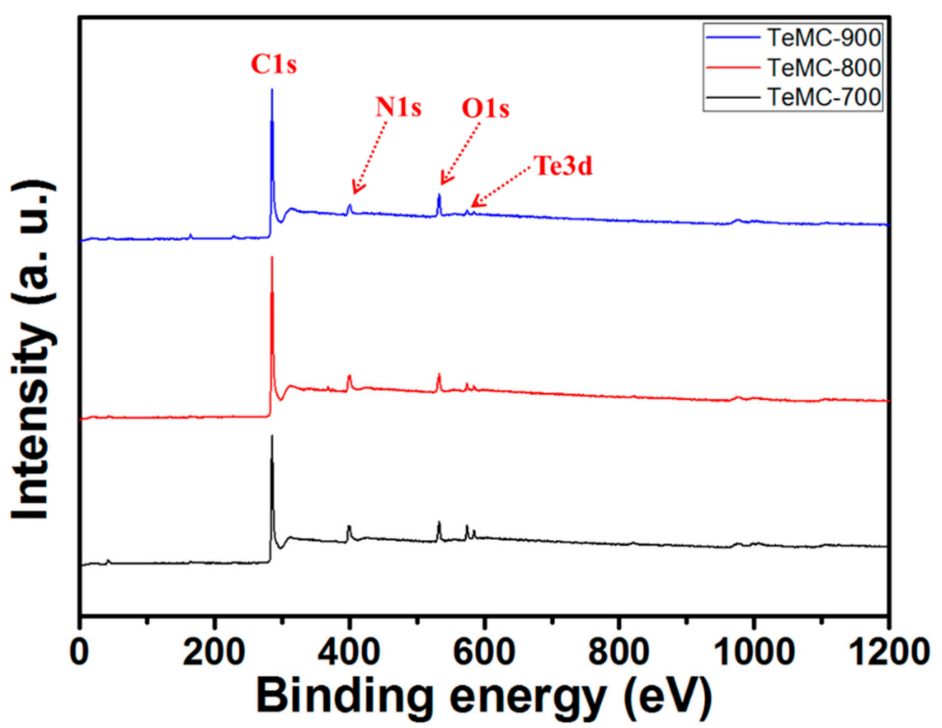

Figure 5. X-ray photoelectron spectroscopy spectra of TeMC materials with different carbonization temperatures.

Table 2. Elemental composition as determined by x-ray photoelectron spectroscopy.

\begin{tabular}{ccccc}
\hline \multirow{2}{*}{ Sample } & \multicolumn{4}{c}{ Element (at.\%) } \\
\cline { 2 - 5 } & $\mathbf{C}$ & $\mathbf{O}$ & $\mathbf{N}$ & $\mathbf{T e}$ \\
\hline TeMC-700 & 83.63 & 5.80 & 10.01 & 0.55 \\
\hline TeMC-800 & 86.34 & 5.04 & 8.36 & 0.26 \\
\hline TeMC-900 & 87.47 & 6.40 & 5.73 & 0.21 \\
\hline
\end{tabular}

Before evaluating the electrochemical performance, TeMC electrodes were prepared by a simple electrospray method. The preparation method was as follows (see Figure 6). First, the as-prepared TeMC materials were homogeneously dispersed in an isopropyl alcohol solution by ultrasonication. Then, this dispersed solution was centrifuged to remove large particles of TeMC materials. The resultant solution was loaded into a syringe with a stainless-steel needle. A high voltage was applied between the needle and substrate. The TeMC materials were directly deposited on the fluorine-doped $\mathrm{SnO}_{2}$ (FTO)/glass surface. The loaded amount of the TeMC material was controlled by the electrospray time of the dispersed solution. The preparation method of the electrode is described in more detail in the Supporting Information. Generally, the amount of carbon material in the CE greatly affects the catalytic 
performance, because the active site that can contact the electrolyte has changed. Therefore, to confirm the catalytic performance as a function of the loaded amount of the TeMC material, TeMC electrodes with various loaded amounts $(0.1,0.2,0.3$, and $0.5 \mathrm{~mL})$ were prepared by controlling the electrospray time. The prepared electrodes were denoted as TeMC-X (where TeMC is the tellurium-doped, mesoporous carbon, and $X$ is the loaded amount of TeMC materials).

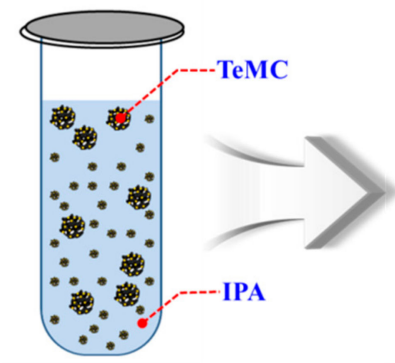

TeMC dispersed solution

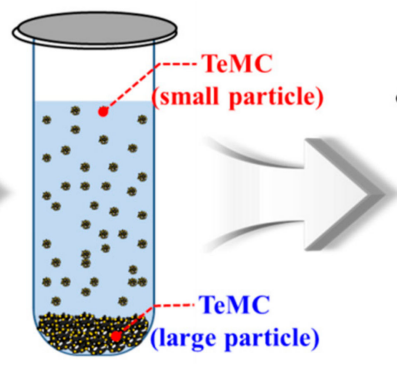

Centrifugation

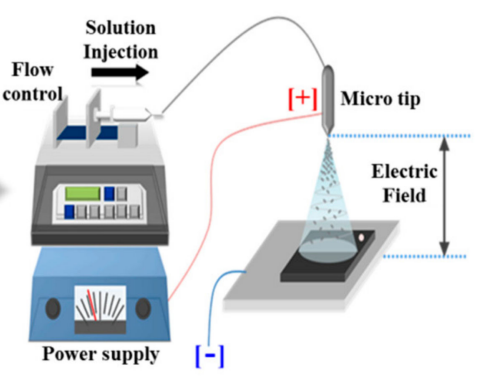

Electrospray

Figure 6. Preparation method of TeMC electrodes via the electrospray technique.

To confirm the surface morphology and coverage of the prepared electrodes, SEM measurements were carried out. Figure 7 shows SEM images of the pristine FTO glass, the Pt electrode, and the TeMC electrodes (with the various loaded amounts of TeMC materials). As shown in the SEM image, the platinum nanoparticles of the Pt electrode are uniformly distributed on the FTO glass surface, which can be seen in Figure 7a,b. Figure 7c-f shows the SEM images of the TeMC electrode surfaces. When the amount of sprayed solution increased from 0.1 to $0.5 \mathrm{~mL}$, the amount of TeMC sprayed on the FTO glass also increased, resulting in increased coverage (See Figure S5).
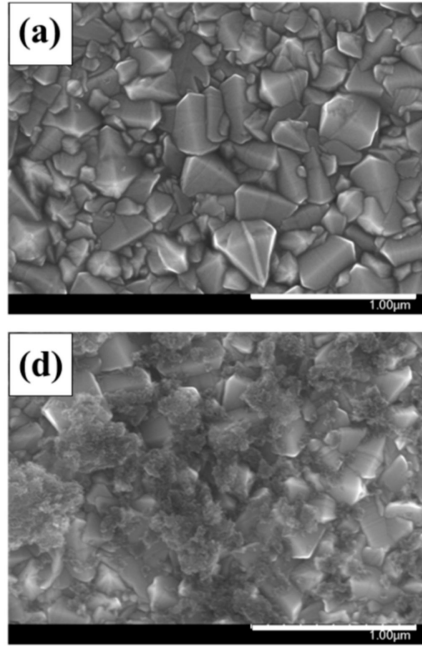
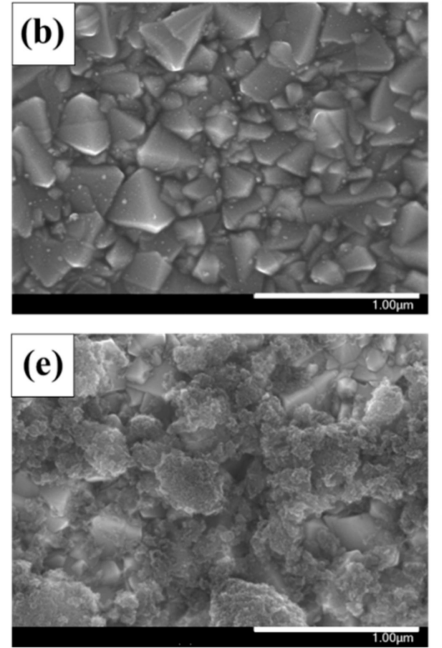
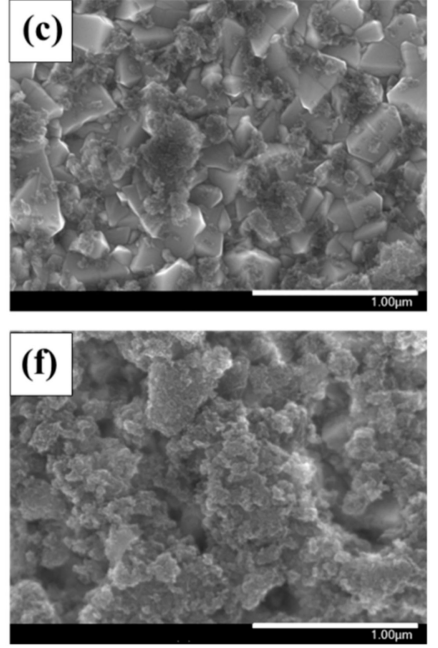

Figure 7. Scanning electron microscopy images of the pristine FTO glass (a), Pt electrode (b), TeMC electrodes (c): TeMC-0.1, (d): TeMC-0.2, (e): TeMC-0.3, (f): TeMC-0.5.

The optical transmittance of the CE is an important factor for bifacial DSSC devices. In addition, the light absorption of the electrolyte is important because illuminated light from the rear side passes through the $\mathrm{CE}$ and the electrolyte before finally reaching the photoanode. Therefore, not only the transmittance of the $\mathrm{CE}$, but also the light absorption of the electrolyte, should be considered for enhancing the performance of bifacial DSSC devices. To confirm the transparency of Pt and TeMC CEs, visible transmittance spectra were obtained. Figure 8a shows the transmittance spectra of pristine FTO glass, the Pt CE, and TeMC CEs with different loading amounts (TeMC material was carbonized at 900 
$\left.{ }^{\circ} \mathrm{C}\right)$. The FTO glass has a transmittance of about $80 \%$ in the visible light region $(400-800 \mathrm{~nm})$. The Pt $\mathrm{CE}$, as a reference, was prepared by a thermal decomposition method; the transmittance was around $75 \%$. In the case of the as-prepared TeMC CEs, the transmittance was lower than that of the Pt CE; as the loaded amount increased, the transmittance decreased significantly. TeMC-0.1 CE, which showed the highest transmittance, exhibited a high transmittance, i.e., about 70\%, in the visible light region. As mentioned above, the performance of bifacial DSSCs largely depends on the transmittance of the CE, as well as the light absorption in the electrolyte. Figure $8 \mathrm{~b}$ shows the absorbance spectra of the cobalt electrolyte. The cobalt electrolyte significantly absorbs light below the wavelength of $400 \mathrm{~nm}$.
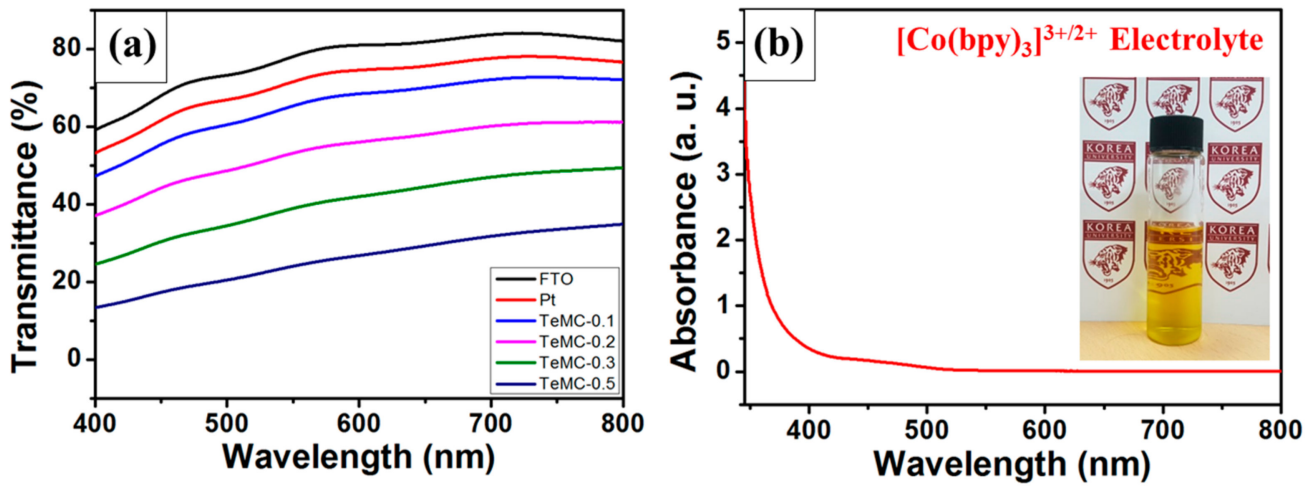

Figure 8. (a) Transmittance spectra of pristine FTO glass, the Pt CE, and the TeMC CEs (different loading amounts of TeMC materials). (b) Absorption spectra of the cobalt electrolyte, which was diluted 100 times.

To evaluate the electrocatalytic ability of CEs for the cobalt redox mediator $\left[\mathrm{Co}(\mathrm{bpy}) 3^{2+/ 3+}\right], \mathrm{Pt}$ and TeMC CEs were prepared by thermal decomposition and electrospray methods. The symmetrical dummy cells (see Figure S6), with two identical electrodes, were fabricated in order to evaluate the electrode performance. Electrochemical impedance spectroscopy (EIS) is widely used in various fields; it is one of the most useful methods for evaluating electrode performance. First, to optimize the TeMC CEs (different carbonization temperatures), the loaded amount of each TeMC material was fixed (0.5 mL sprayed solution), and symmetrical dummy cells were fabricated. Figure S7a shows the EIS data for TeMC CEs with different carbonization temperatures. There are two different semicircles with charge transfer resistances $\left(R_{c t}\right)$ in the high-frequency region and Nernst diffusion impedances $\left(Z_{N}\right)$ in the low-frequency region. The $R_{c t}$ is related to the charge transfer resistance at the CE/electrolyte interface, and $\mathrm{Z}_{\mathrm{N}}$ is the diffusion resistance of the electrolyte between the electrodes (corresponding equivalent circuit, Figure S6). Figure S7a shows the EIS results of all TeMC CEs (carbonization temperatures of 700,800 , and $900^{\circ} \mathrm{C}$ ), exhibiting a lower $R_{c t}$ value than the Pt CE. Among them, the TeMC CE manufactured at $900^{\circ} \mathrm{C}$ had the smallest $R_{c t}$ value. According to the above results (XPS, Raman, and electrical conductivity), the TeMC-900 material has a low Te doping level and few defect sites, but it has high electrical conductivity. Generally, the CE requires good electrocatalytic properties as well as high electrical conductivity to facilitate the movement of electrons from the external circuit to the electrode surface. For these reasons, the TeMC-900 CE showed better electrocatalytic properties than other TeMC CEs. Thereafter, to make the transparent electrode, the amount of loaded TeMC-900 on the FTO glass was optimized by controlling the electrospray time. When the loaded amount was decreased from 0.5 to $0.1 \mathrm{~mL}$ of the dispersed solution of the TeMC-900 material, the $R_{c t}$ value increased. In the case of $0.08 \mathrm{~mL}$, it had a larger $R_{c t}$ value than the Pt CE. Thereafter, the electrodes were prepared under these optimized conditions (carbonization temperature: $900^{\circ} \mathrm{C}$, amount of sprayed TeMC solution: $0.1 \mathrm{~mL}$ ), and were denoted as the TeMC. The role of the CE is to regenerate the oxidized redox species in the electrolyte. The rate of charge transfer from the $\mathrm{CE}$ to the electrolyte affects the cathode performance in the DSSC, and can also affect photocurrent generation in the photoanode through dye regeneration by the redox mediator. Therefore, the regeneration rate of the redox mediator by the CE should be 
comparable to the rate of dye regeneration. The electrocatalytic performance of the CE is determined by the charge transfer resistance $\left(R_{c t}\right)$, which is related to the exchange current density $\left(J_{0}\right)$, as in the following equation (1) [38]:

$$
J_{0}=R T / n F R_{c t}
$$

where $R, T, n, F$, and $R_{c t}$ are the gas constant, temperature, number of electrons involved in the electrochemical reaction of the redox mediator, Faraday's constant, and charge transfer resistance, respectively. The higher the $J_{0}$ value, the better the electrocatalytic ability of the CE. Figure 9 shows the EIS and Tafel-polarization plots of the Pt and TeMC dummy cells, and the corresponding $J_{0}$ values are summarized in Table 3. The $R_{c t}$ value of TeMC is $0.49 \Omega \mathrm{cm}^{2}$, which is lower than that of $\mathrm{Pt}(0.62 \Omega$ $\mathrm{cm}^{2}$ ), with corresponding $J_{0}$ values of 52.43 and $41.44 \mathrm{~mA} \mathrm{~cm}^{-2}$, respectively. The TeMC CE prepared under optimized conditions showed better electrocatalytic properties than the Pt CE, and it exhibited a transmittance of over $70 \%$ in the visible light region (See Figure 8a). It is possible to fabricate bifacial DSSC devices because of these features. In addition, the good electrocatalytic ability of the TeMC CE can be confirmed from the Tafel-polarization data. The value of $J_{0}$ is lower than that of the $J_{0}$ value calculated from EIS data, but the tendency is well-matched.
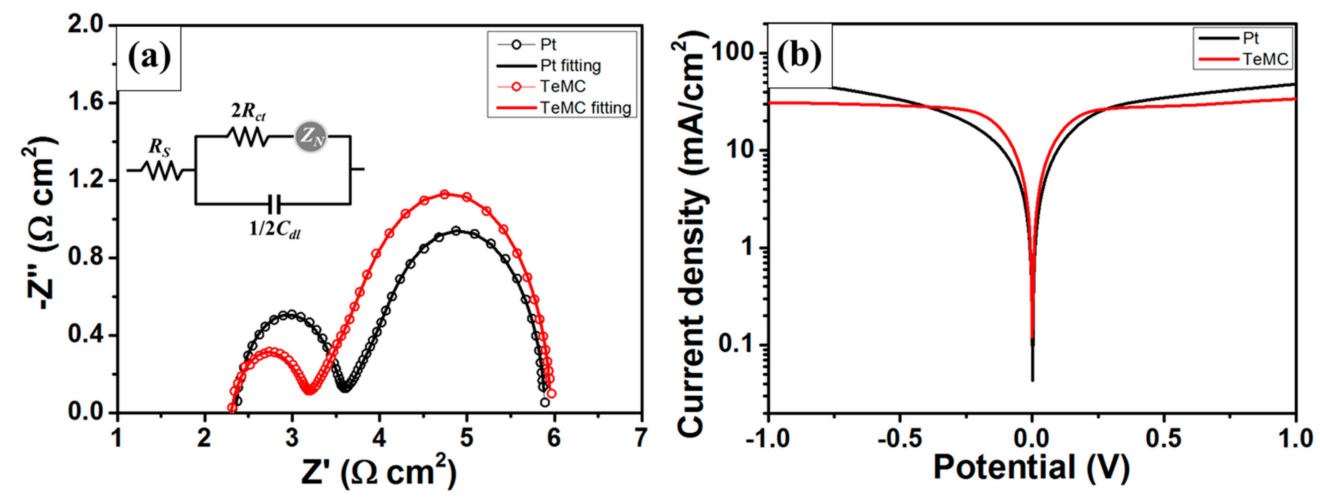

Figure 9. (a) Nyquist plots and (b) Tafel-polarization plots of the Pt and TeMC dummy cells with the $\mathrm{Co}(\mathrm{bpy}) 3^{2+/ 3+}$ electrolyte.

Table 3. Electrochemical parameters of the CE in the symmetrical dummy cells by LSV (linear sweep voltammetry) and EIS (electrochemical impedance specteoscopy).

\begin{tabular}{|c|c|c|c|c|c|c|c|}
\hline \multirow[b]{2}{*}{ CE } & \multirow[b]{2}{*}{ Electrolyte } & \multicolumn{2}{|c|}{ LSV } & \multicolumn{4}{|c|}{ EIS } \\
\hline & & $\begin{array}{c}R_{C V} \\
\left(\Omega \mathrm{cm}^{2}\right)\end{array}$ & $\begin{array}{c}J_{o} \\
\left(\mathrm{~mA} \mathrm{~cm}^{-2}\right)\end{array}$ & $\begin{array}{c}R_{S} \\
\left(\Omega \mathrm{cm}^{2}\right)\end{array}$ & $\begin{array}{c}R_{c t} \\
\left(\Omega \mathrm{cm}^{2}\right)\end{array}$ & $\begin{array}{c}C_{d l} \\
\left.(\mu \mathrm{F} \mathrm{cm})^{2}\right)\end{array}$ & $\begin{array}{c}J_{o} \\
\left(\mathrm{~mA} \mathrm{~cm}^{-2}\right)\end{array}$ \\
\hline $\mathrm{Pt}$ & Co(bnu) 2+/3+ & 9.37 & 2.74 & 2.30 & 0.62 & 14.38 & 41.44 \\
\hline TeMC & & 6.87 & 3.74 & 2.17 & 0.49 & 28.91 & 52.43 \\
\hline
\end{tabular}

To further investigate the electrocatalytic activity of the TeMC CE for the cobalt electrolyte, cyclic voltammetry (CV) was used, and the Pt CE was also studied for performance comparison. As shown in Figure 10, two pairs of redox couples, corresponding to the anodic peak current $\left(I_{p a}\right)$ and cathodic peak current $\left(I_{p c}\right)$, were observed in both Pt and TeMC CEs. These $I_{p a}$ and $I_{p c}$ peaks are related to the oxidation of $\mathrm{Co}(\mathrm{bpy}) 3^{2+}$ and the reduction of $\mathrm{Co}(\mathrm{bpy})_{3}{ }^{3+}$ ions, respectively. When evaluating the electrocatalytic ability by CV measurements, the peak current and peak-to-peak distance $\left(E_{p p}\right)$ are important parameters. If the $\mathrm{CE}$ has a higher peak current and narrow $E_{p p}$ values, it has excellent electrocatalytic ability. Even though the Pt and TeMC CEs have similar peak currents, the TeMC CE exhibits good electrocatalytic ability because it has a narrower $E_{p p}$ value than the $\mathrm{Pt} \mathrm{CE}$. The same trend was observed for the CV curves measured at different scan rates (See Figure S8). This CV result was well-matched with the results of the other electrochemical analyses mentioned above. 


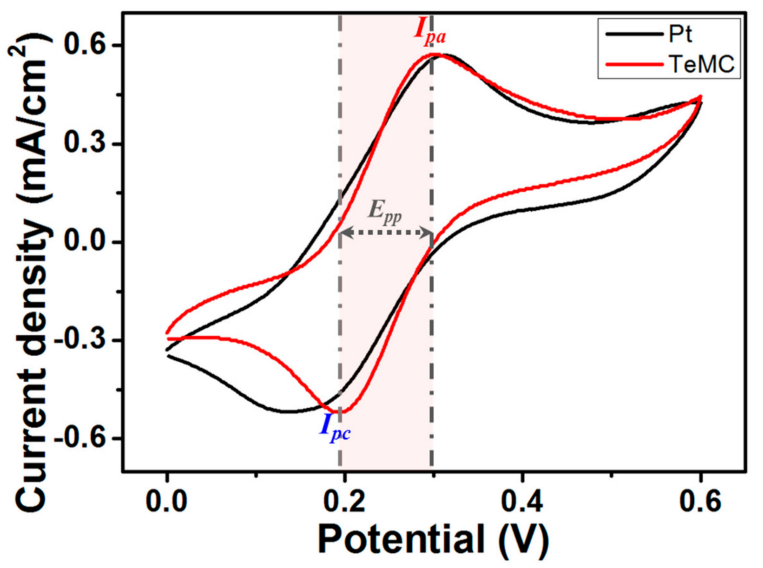

Figure 10. Cyclic voltammetry curves for the $\mathrm{Co}(\mathrm{bpy})_{3}{ }^{2+/ 3+}$ redox couples obtained at a scan rate of $50 \mathrm{mV} \mathrm{s}^{-1}$ using the $\mathrm{Pt}$ and TeMC CEs as the working electrode, a Pt wire as the $\mathrm{CE}, \mathrm{Ag} / \mathrm{AgCl}$ as the reference electrode, and $0.1 \mathrm{M} \mathrm{LiClO}_{4}$ as the supporting electrolyte.

Based on the electrochemical analysis and various in-depth evaluations of TeMC CEs, the electrocatalytic ability of TeMC CEs was evaluated in an actual DSSC device employing the SM315 [39] sensitizer and the cobalt electrolyte $\left[\mathrm{Co}(\mathrm{bpy})_{3}{ }^{2+/ 3+}\right]$. Before fabricating the bifacial devices, we first confirmed the current-voltage (J-V) performance of the DSSC with the different TeMC CEs loading amounts. The fabrication method of the DSSC device is precisely described in the Supporting Information. Figure S9 shows the J-V curves of SM315/ Co(bpy $)_{3}{ }^{2+/ 3+}$ based DSSC devices with Pt and TeMC CEs. The corresponding incident photon-to-current conversion efficiency (IPCE) data of DSSC devices and their photovoltaic performances are summarized in Table S1. In the dummy cell test, all TeMC CEs (except for TeMC-0.08 CE) had smaller $R_{c t}$ values than the Pt CE, and the $R_{c t}$ value increased by reducing the loaded amount of the TeMC material (See Figure S10; In the case of full cell devices, the TeMC-based device has a smaller $R_{c t}$ value than that of Pt-based device.). Although the $R_{c t}$ value increased, it could already meet the current generated from the photoanode. Therefore, all devices have similar PCE values (Pt: 12.01\%, TeMC-0.1: 12.07\%, TeMC-0.2: 12.10\%, TeMC-0.3: 12.13\%, TeMC-0.5: $12.17 \%$ ) when fabricating the actual DSSC device (with scattering layer).

To confirm the performance of the bifacial DSSC device, $\mathrm{Pt}$ and TeMC CEs were applied to the DSSC device without a scattering layer. Figure 11 shows the J-V curves of bifacial DSSC devices employing the Pt and TeMC CEs and the IPCE data. The detailed photovoltaic performances are displayed in Table 4. The bifacial DSSC devices (see Figure 11a inset) can absorb both incoming light from the front (front irradiation) and rear (rear irradiation) side. Therefore, to use most of the light coming from the rear side, the CE must be transparent, and the electrolyte must absorb the small amount of light. In the case of DSSC performance for front irradiation, the TeMC CE-based device $(9.43 \%)$ showed a higher power conversion efficiency (PCE) than the Pt CE (9.23\%). As shown in Table 4, the short circuit current (JSC; Pt: $14.58 \mathrm{~mA} \mathrm{~cm}{ }^{-2}$, TeMC: $14.48 \mathrm{~mA} \mathrm{~cm}{ }^{-2}$ ) and open circuit voltage ( $V_{O C}$; Pt: $820.51 \mathrm{mV}$, TeMC: $822.14 \mathrm{mV}$ ) values of both devices are similar, but the fill factor (FF; Pt: 77.13\%, TeMC: 79.23\%) values are somewhat different due to the difference in electrocatalytic ability. When irradiated from the rear side, both devices employing Pt and TeMC CEs showed lower PCE values than front irradiated efficiency. The reason for the decrease in the PCE is the low $J_{S C}$ value, which occurred because light below a wavelength of $500 \mathrm{~nm}$ is almost absorbed in the cobalt electrolyte when it is irradiated from the rear side (see Figure 8b). Because of this, the IPCE results (Figure 11b) also showed low conversion efficiency at wavelengths below $500 \mathrm{~nm}$; the light is absorbed in not only the electrolyte, but also the CE. As shown in Figure 8a, Pt and TeMC CEs exhibited transmittances of about 75\% and 70\%, respectively. In the case of full cells, Pt and TeMC CE-based DSSCs (see Figure S11) gave average transmittances of about $30.76 \%$ and $26.53 \%$, respectively. Therefore, the TeMC $(8.06 \%)$ electrode, with relatively low transmittance, showed a slightly lower PCE performance than the Pt 
$(8.42 \%)$ electrode. This is a result of the difference in the amount of light reaching the photoanode, depending on the transmittance of the CEs. Although the TeMC CE had slightly lower rear side irradiation performance than the Pt CE, it showed good performance in both side irradiations due to its high transmittance and electrocatalytic properties.
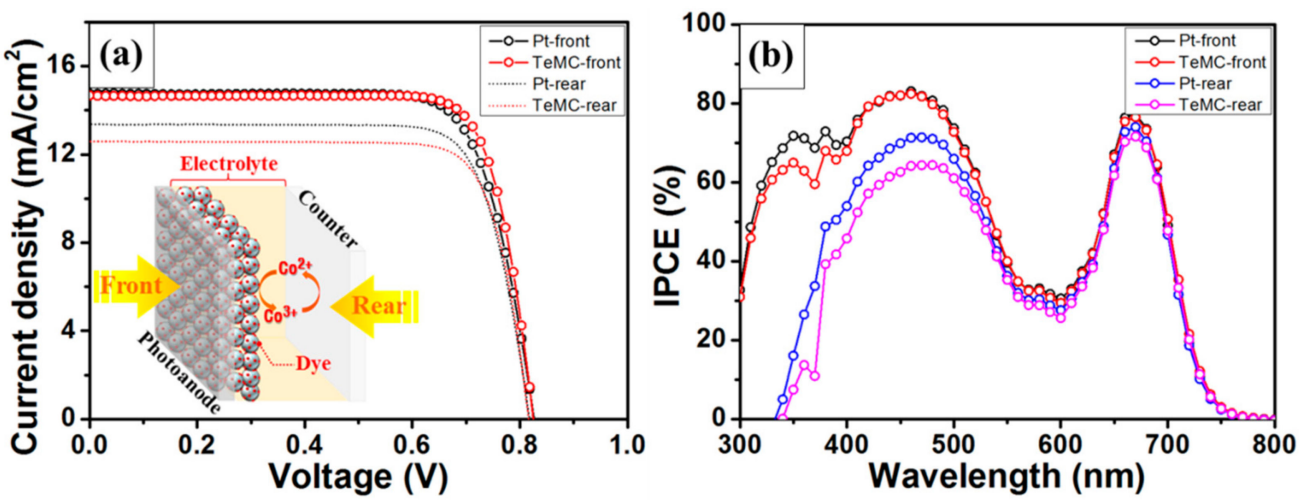

Figure 11. (a) $J-V$ curves of bifacial DSSC devices with Pt and TeMC CEs under one sun illumination (AM $1.5 \mathrm{G}$ ). The inset is a schematic illustration of the bifacial DSSC device. (b) The corresponding IPCE data of the bifacial DSSC devices.

The electrochemical stability of the $\mathrm{CE}$ is one of the most important factors for the practical use of DSSC devices. To confirm the electrochemical stability of the cobalt electrolyte, the dummy cells with Pt and TeMC CEs were freshly prepared. Then, the $R_{c t}$ value was confirmed by EIS analysis, measured at $0 \mathrm{~V}$ from $10^{6}$ to $10^{-1} \mathrm{~Hz}$. After measuring the $R_{c t}$ value, the dummy cells were tested through 50 consecutive potential CV cycles from -1 to $1 \mathrm{~V}$ at a scan rate of $50 \mathrm{mV} \mathrm{s}^{-1}$. These EIS and $\mathrm{CV}$ tests were repeated 10 times to confirm the electrochemical stability of the CEs. Figure 12 shows the electrochemical stability test of the symmetrical dummy cells with Pt and TeMC CEs. As shown in Figure 12, the $R_{c t}$ value of the Pt CE increased significantly from the first to the final cycle. On the other hand, the $R_{c t}$ value of the TeMC CE increased slightly. Thus, it can be concluded that the TeMC CE has better electrochemical stability than the Pt CE in cobalt electrolytes.
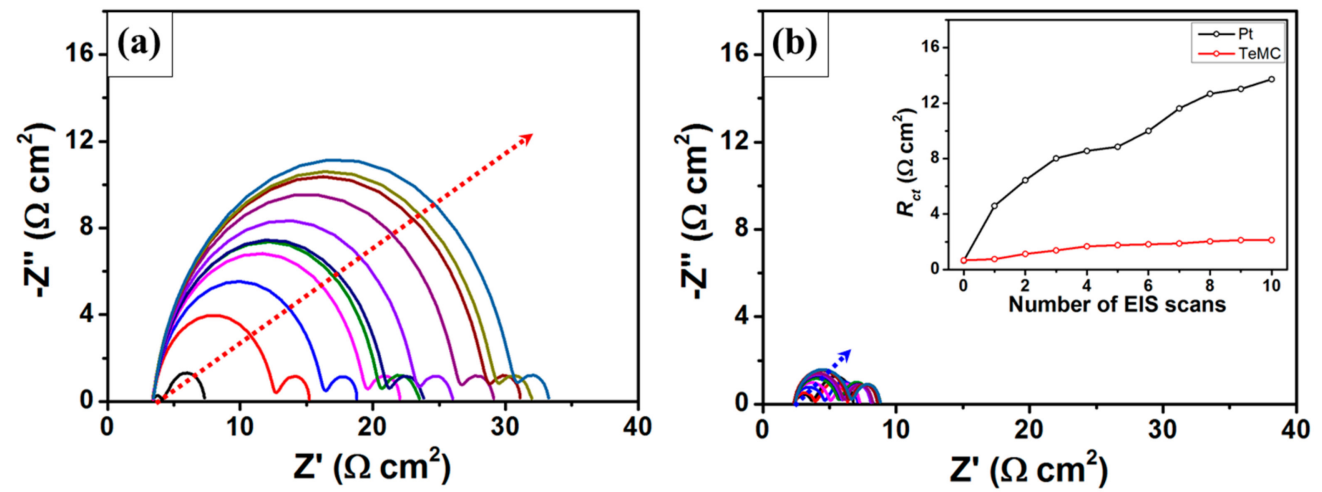

Figure 12. Electrochemical stability test of symmetrical dummy cells with $\mathrm{Pt}(\mathbf{a})$ and TeMC CEs (b). The sequence of measurements is as follows: EIS was measured at $0 \mathrm{~V}$ from $10^{6}$ to $10^{-1} \mathrm{~Hz}$. Then, CV scans of 50 cycles were performed from -1 to $1 \mathrm{~V}$ at a scan rate of $50 \mathrm{mV} \mathrm{s}^{-1}$. The sequence of the electrochemical test was repeated 10 times. The inset is the $R_{c t}$ value change of dummy cells with $\mathrm{Pt}$ and TeMC CEs. 
Table 4. Photovoltaic performance of DSSCs.

\begin{tabular}{cccccc}
\hline Sample & Dye/Electrolyte & $J_{S C}\left(\mathbf{m A ~ c m}^{-\mathbf{2}}\right)$ & $V_{\text {OC }}(\mathbf{m V})$ & $\boldsymbol{F F}(\mathbf{\%})$ & PCE $(\%) *$ \\
\hline Pt-front & & $14.58 \pm 0.11$ & $820.51 \pm 4.86$ & $77.13 \pm 0.57$ & $9.23 \pm 0.07$ \\
TeMC-front & & $14.48 \pm 0.12$ & $822.14 \pm 3.73$ & $79.23 \pm 0.23$ & $9.43 \pm 0.10$ \\
Pt-rear & & $13.25 \pm 0.14$ & $817.00 \pm 1.43$ & $77.78 \pm 0.10$ & $8.42 \pm 0.08$ \\
TeMC-rear & & $12.52 \pm 0.11$ & $813.38 \pm 3.04$ & $79.13 \pm 0.25$ & $8.06 \pm 0.09$ \\
\hline
\end{tabular}

* The average power conversion efficiency (PCE) was calculated in five different cells, measured under AM 1.5 G illumination $\left(100 \mathrm{~mW} \mathrm{~cm}^{-2}\right)$.

\section{Conclusions}

In summary, a transparent CE for bifacial DSSC devices was prepared using TeMC material. The TeMC had a high doping level (tellurium: 0.21 at.\% and nitrogen: 5.74 at.\%, from XPS study) and consisted mainly of a mesoporous structure with high electrical conductivity. The TeMC CE itself exhibited high transparency, i.e., about 70\% (FTO glass: about 80\%, Pt CE: about 75\%), while maintaining high degree of electrocatalytic activity and electrical conductivity, as well as improved electrochemical stability. When applied to the bifacial DSSC device, the efficiency of the rear side (TeMC: 8.06\%, Pt: 8.42\%) was slightly lower than that of the Pt CE, because the transmittance was slightly lower than that of $\mathrm{Pt}$, but the front side (TeMC: $9.43 \%$, Pt: 9.23\%) showed better performance due to the good electrocatalytic properties. Based on our results, newly-developed transparent, carbon-based electrodes led to a more stable and effective bifacial DSSC without sacrificing the photovoltaic performance of the device. This technology can be applied to various fields, such as batteries, fuel cells, sensors, etc.

Supplementary Materials: The following are available online at http://www.mdpi.com/2079-4991/10/1/29/s1, Figure S1: Electrical conductivity data of TeMC materials with different carbonization temperatures, Figure S2: High-resolution XPS spectra of TeMC materials with different carbonization temperatures, Figure S3: Bonding distributions of nitrogen in TeMC materials, Figure S4: (a) SEM image and (b-e) corresponding elemental mappings of TeMC materials, Figure S5: Digital photograph images of (a) FTO glass, (b) the Pt CE, and (c-f) TeMC CEs, Figure S6: (a) Symmetrical dummy cell and (b) the equivalent circuit, Figure S7: Nyquist plot of Pt and TeMC CEs with (a) different carbonization temperatures and (b) various loaded amounts of TeMC materials, Figure S8: CV curves of CEs with different scan rates for the cobalt redox couple, Figure S9: (a) J-V curves of SM315/Co(bpy)32+/3+ based DSSC devices with Pt and TeMC CEs (with different loaded amounts of the TeMC CE) under one sun illumination (AM 1.5 G). (b) The corresponding IPCE data of DSSC devices, Figure S10: (a) Expended impedance spectra of the high-frequency region for SM315/Co(bpy)32+/3+ based DSSC devices employing Pt and TeMC CEs. (b) Bode plots obtained with the same devices, Figure S11: Transmittances spectra of the DSSC devices with Pt and TeMC CEs, Table S1: Photovoltaic performance of DSSCs with Pt and TeMC CEs, Table S2: Comparison of reported performances in bifacial DSSCs.

Author Contributions: C.K.K.: Synthesis, DSSC fabrication, DSSC measurements and manuscript preparation; J.-M.J.: contribution to DSSC fabrication; H.Z.: contribution to manuscript preparation and supervision; C.L.: contribution to DSSC measurements; H.K.K.: project leader, concepts and contribution to manuscript preparation. All authors have read and agreed to the published version of the manuscript.

Funding: This work was funded by the Korean government (Ministry of Science, ICT and Future Planning) through the Mid-career Researcher Program (NRF-2017R1A2A1A17069374), and the Human Resources Program in Energy Technology of the Korea Institute of Energy Technology Evaluation and Planning (KETEP) granted financial resources from the Ministry of Trade, Industry \& Energy, Republic of Korea (KETEP-20184030201910).

Conflicts of Interest: The authors declare no conflict of interest.

\section{References}

1. Oregan, B.; Gratzel, M. A Low-Cost, High-Efficiency Solar-Cell Based on Dye-Sensitized Colloidal TiO 2 Films. Nature 1991, 353, 737-740. [CrossRef]

2. Grätzel, M. Photoelectrochemical cells. Nature 2001, 414, 338.

3. Mathew, S.; Yella, A.; Gao, P.; Humphry-Baker, R.; Curchod, B.F.; Ashari-Astani, N.; Tavernelli, I.; Rothlisberger, U.; Nazeeruddin, M.K.; Grätzel, M. Dye-sensitized solar cells with $13 \%$ efficiency achieved through the molecular engineering of porphyrin sensitizers. Nat. Chem. 2014, 6, 242-247.

4. Ji, J.M.; Zhou, H.; Kim, H.K. Rational design criteria for D- $\pi-A$ structured organic and porphyrin sensitizers for highly efficient dye-sensitized solar cells. J. Mater. Chem. A 2018, 6, 14518-14545. [CrossRef] 
5. Freitag, M.; Teuscher, J.; Saygili, Y.; Zhang, X.; Giordano, F.; Liska, P.; Hua, J.; Zakeeruddin, S.M.; Moser, J.-E.; Grätzel, M.; et al. Dye-sensitized solar cells for efficient power generation under ambient lighting. Nat. Photonics 2017, 11, 372. [CrossRef]

6. Saifullah, M.; Gwak, J.; Yun, J.H. Comprehensive review on material requirements, present status, and future prospects for building-integrated semitransparent photovoltaics (BISTPV). J. Mater. Chem. A 2016, 4, 8512-8540. [CrossRef]

7. Yuan, H.; Wang, W.; Xu, D.; Xu, Q.; Xie, J.; Chen, X.; Zhang, T.; Xiong, C.; He, Y.; Zhang, Y.; et al. Outdoor testing and ageing of dye-sensitized solar cells for building integrated photovoltaics. Sol. Energy 2018, 165, 233-239. [CrossRef]

8. Xie, Y.; Tang, Y.; Wu, W.; Wang, Y.; Liu, J.; Li, X.; Tian, H.; Zhu, W.-H. Porphyrin Cosensitization for a Photovoltaic Efficiency of 11.5\%: A Record for Non-Ruthenium Solar Cells Based on Iodine Electrolyte. J. Am. Chem. Soc. 2015, 137, 14055-14058. [CrossRef]

9. Zhou, H.; Ji, J.-M.; Kang, S.H.; Kim, M.S.; Lee, H.S.; Kim, C.H.; Kim, H.K. Molecular design and synthesis of $\mathrm{D}-\pi-\mathrm{A}$ structured porphyrin dyes with various acceptor units for dye-sensitized solar cells. J. Mater. Chem. C 2019, 7, 2843-2852. [CrossRef]

10. Zhou, H.; Ji, J.-M.; Kim, M.S.; Kim, H.K. Significant Influence of a Single Atom Change in Auxiliary Acceptor on Photovoltaic Properties of Porphyrin-Based Dye-Sensitized Solar Cells. Nanomater. 2018, 8, 1030. [CrossRef]

11. Yun, S.; Hagfeldt, A.; Ma, T. Pt-free counter electrode for dye-sensitized solar cells with high efficiency. Adv. Mater. 2014, 26, 6210-6237.

12. Thomas, S.; Deepak, T.G.; Anjusree, G.S.; Arun, T.A.; Nair, S.V.; Nair, A.S. A review on counter electrode materials in dye-sensitized solar cells. J. Mater. Chem. A 2014, 2, 4474-4490. [CrossRef]

13. Wu, J.; Lan, Z.; Lin, J.; Huang, M.; Huang, Y.; Fan, L.; Luo, G.; Lin, Y.; Xie, Y.; Wei, Y. Counter electrodes in dye-sensitized solar cells. Chem. Soc. Rev. 2017, 46, 5975-6023. [CrossRef] [PubMed]

14. Xue, Y.; Liu, J.; Chen, H.; Wang, R.; Li, D.; Qu, J.; Dai, L. Nitrogen-Doped Graphene Foams as Metal-Free Counter Electrodes in High-Performance Dye-Sensitized Solar Cells. Angew. Chem. 2012, 124, 12290-12293. [CrossRef]

15. Bu, C.; Liu, Y.; Yu, Z.; You, S.; Huang, N.; Liang, L.; Zhao, X.-Z. Highly Transparent Carbon Counter Electrode Prepared via an in Situ Carbonization Method for Bifacial Dye-Sensitized Solar Cells. ACS Appl. Mater. Interfaces 2013, 5, 7432-7438. [CrossRef]

16. Jung, S.-M.; Choi, I.T.; Lim, K.; Ko, J.; Kim, J.C.; Lee, J.-J.; Ju, M.J.; Kim, H.K.; Baek, J.-B. B-Doped Graphene as an Electrochemically Superior Metal-Free Cathode Material As Compared to Pt over a Co(II)/Co(III) Electrolyte for Dye-Sensitized Solar Cell. Chem. Mater. 2014, 26, 3586-3591. [CrossRef]

17. Nemala, S.S.; Aneja, K.S.; Bhargava, P.; Bohm, H.M.; Mallick, S.; Bohm, S. Novel high-pressure airless spray exfoliation method for graphene nanoplatelets as a stable counter electrode in DSSC. Electrochimica Acta 2018, 285, 86-93. [CrossRef]

18. Cho, S.; Hwang, S.H.; Kim, C.; Jang, J. Polyaniline porous counter-electrodes for high performance dye-sensitized solar cells. J. Mater. Chem. 2012, 22, 12164-12171. [CrossRef]

19. Wei, W.; Wang, H.; Hu, Y.H. A review on PEDOT-based counter electrodes for dye-sensitized solar cells. Int. J. Energy Res. 2014, 38, 1099-1111. [CrossRef]

20. Khan, A.; Huang, Y.-T.; Miyasaka, T.; Ikegami, M.; Feng, S.-P.; Li, W.-D. Solution-Processed Transparent Nickel-Mesh Counter Electrode with in-Situ Electrodeposited Platinum Nanoparticles for Full-Plastic Bifacial Dye-Sensitized Solar Cells. ACS Appl. Mater. Interfaces 2017, 9, 8083-8091. [CrossRef]

21. Chang, P.-J.; Cheng, K.-Y.; Chou, S.-W.; Shyue, J.-J.; Yang, Y.-Y.; Hung, C.-Y.; Lin, C.-Y.; Chen, H.-L.; Chou, H.-L.; Chou, P.-T. Tri-iodide Reduction Activity of Shape- and Composition-Controlled PtFe Nanostructures as Counter Electrodes in Dye-Sensitized Solar Cells. Chem. Mater. 2016, 28, 2110-2119. [CrossRef]

22. Li, Y.; Feng, Q.; Wang, H.; Zhou, G.; Wang, Z.S. Reduced graphene oxide-Ta3N5 composite: A potential cathode for efficient $\mathrm{Co}(\mathrm{bpy})_{3}{ }^{2+/ 3+}$ mediated dye-sensitized solar cells. J. Mater. Chem. A. 2013, 1, 6342-6349.

23. Ramasamy, E.; Jo, C.; Anthonysamy, A.; Jeong, I.; Kim, J.K.; Lee, J. Soft-Template Simple Synthesis of Ordered Mesoporous Titanium Nitride-Carbon Nanocomposite for High Performance Dye-Sensitized Solar Cell Counter Electrodes. Chem. Mater. 2012, 24, 1575-1582. [CrossRef] 
24. Ju, M.J.; Lim, K.; Mohin, J.; Kim, H.K.; Choi, I.T.; Zhong, M.; Ko, J.; Lamson, M.; Kowalewski, T.; Matyjaszewski, K. Copolymer-templated nitrogen-enriched nanocarbons as a low charge-transfer resistance and highly stable alternative to platinum cathodes in dye-sensitized solar cells. J. Mater. Chem. A 2015, 3, 4413-4419. [CrossRef]

25. Ju, M.J.; Jeon, I.-Y.; Kim, H.M.; Choi, J.I.; Jung, S.-M.; Seo, J.-M.; Choi, I.T.; Kang, S.H.; Kim, H.S.; Noh, M.J.; et al. Edge-selenated graphene nanoplatelets as durable metal-free catalysts for iodine reduction reaction in dye-sensitized solar cells. Sci. Adv. 2016, 2, e1501459. [CrossRef]

26. Guo, N.; Shibuya, R.; Akiba, C.; Saji, S.; Kondo, T.; Nakamura, J. Active sites of nitrogen-doped carbon materials for oxygen reduction reaction clarified using model catalysts. Sci. 2016, 351, 361-365. [CrossRef]

27. Frackowiak, E. Carbon materials for supercapacitor application. Phys. Chem. Chem. Phys. 2007, 9, 1774. [CrossRef]

28. Roberts, A.D.; Li, X.; Zhang, H. Porous carbon spheres and monoliths: morphology control, pore size tuning and their applications as Li-ion battery anode materials. Chem. Soc. Rev. 2014, 43, 4341-4356. [CrossRef]

29. Baptista, F.R.; Belhout, S.A.; Giordani, S.; Quinn, S.J. Recent developments in carbon nanomaterial sensors. Chem. Soc. Rev. 2015, 44, 4433-4453. [CrossRef]

30. Kim, C.K.; Zhou, H.; Kowalewski, T.; Matyjaszewski, K.; Kim, H.K. Soft-templated tellurium-doped mesoporous carbon as a Pt-free electrocatalyst for high-performance dye-sensitized solar cells. ACS Appl. Mater. Inter. 2019, 11, 2093-2102.

31. Duan, Y.; Tang, Q.; Liu, J.; He, B.; Yu, L. Transparent Metal Selenide Alloy Counter Electrodes for High-Efficiency Bifacial Dye-Sensitized Solar Cells. Angew. Chem. Int. Ed. 2014, 53, 14569-14574. [CrossRef] [PubMed]

32. Song, D.; Li, M.; Li, Y.; Zhao, X.; Jiang, B.; Jiang, Y. Highly Transparent and Efficient Counter Electrode Using SiO2/PEDOT-PSS Composite for Bifacial Dye-Sensitized Solar Cells. ACS Appl. Mater. Interfaces 2014, 6, 7126-7132. [CrossRef] [PubMed]

33. Kang, J.S.; Kim, J.; Kim, J.-Y.; Lee, M.J.; Kang, J.; Son, Y.J.; Jeong, J.; Park, S.H.; Ko, M.J.; Sung, Y.-E. Highly Efficient Bifacial Dye-Sensitized Solar Cells Employing Polymeric Counter Electrodes. ACS Appl. Mater. Interfaces 2018, 10, 8611-8620. [CrossRef] [PubMed]

34. Leofanti, G.; Padovan, M.; Tozzola, G.; Venturelli, B. Surface area and pore texture of catalysts. Catal. Today 1998, 41, 207-219. [CrossRef]

35. Shang, H.; Lu, Y.; Zhao, F.; Chao, C.; Zhang, B.; Zhang, H. Preparing high surface area porous carbon from biomass by carbonization in a molten salt medium. RSC Adv. 2015, 5, 75728-75734. [CrossRef]

36. Jeon, I.-Y.; Kim, H.M.; Kweon, D.H.; Jung, S.-M.; Seo, J.-M.; Shin, S.-H.; Choi, I.T.; Eom, Y.K.; Kang, S.H.; Kim, H.K.; et al. Metalloid tellurium-doped graphene nanoplatelets as ultimately stable electrocatalysts for cobalt reduction reaction in dye-sensitized solar cells. Nano Energy 2016, 30, 867-876. [CrossRef]

37. Kim, C.K.; Choi, I.T.; Kang, S.H.; Kim, H.K. Anchovy-derived nitrogen and sulfur co-doped porous carbon materials for high-performance supercapacitors and dye-sensitized solar cells. RSC Adv. 2017, 7, 35565-35574. [CrossRef]

38. Hou, Y.; Wang, N.; Yang, X.H.; Fang, W.Q.; Zhang, B.; Wang, H.F.; Lu, G.Z.; Hu, P.; Zhao, H.J.; Yang, H.G. Rational screening low-cost counter electrodes for dye-sensitized solar cells. Nat. Commun. 2013, 4, 1583. [CrossRef]

39. Kang, S.H.; Jeong, M.J.; Eom, Y.K.; Choi, I.T.; Kwon, S.M.; Yoo, Y.J.; Kim, J.; Kwon, J.; Park, J.H.; Kim, H.K. Porphyrin sensitizers with donor structural engineering for superior performance dye-sensitized solar cells and tandem solar cells for water splitting applications. Adv. Energy Mater. 2017, 7, 1602117. [CrossRef]

(C) 2019 by the authors. Licensee MDPI, Basel, Switzerland. This article is an open access article distributed under the terms and conditions of the Creative Commons Attribution (CC BY) license (http://creativecommons.org/licenses/by/4.0/). 